Revue d'histoire de l'Amérique française

REVUE D.HISTOIRE DE L'AMÉRIQUE FRANÇAISE

\title{
Le voyage de La Vérendrye au pays des Mandannes
}

\section{Antoine d'Eschambault}

Volume 2, numéro 3, décembre 1948

URI : https://id.erudit.org/iderudit/801478ar

DOI : https://doi.org/10.7202/801478ar

Aller au sommaire du numéro

\section{Éditeur(s)}

Institut d'histoire de l'Amérique française

\section{ISSN}

0035-2357 (imprimé)

1492-1383 (numérique)

Découvrir la revue

\section{Citer cet article}

d'Eschambault, A. (1948). Le voyage de La Vérendrye au pays des Mandannes. Revue d'histoire de l'Amérique française, 2(3), 424-431.

https://doi.org/10.7202/801478ar d'utilisation que vous pouvez consulter en ligne.

https://apropos.erudit.org/fr/usagers/politique-dutilisation/ 


\section{LE VOYAGE DE LA VERENDRYE AU PAYS DES MANDANNES}

Le voyage de La Vérendrye au pays des Mandannes, ou des Mantannes comme il les appelait invariablement, date de l'automne de 1738. Parti du fort la Reine le 18 octobre il y était de retour le 10 janvier de l'année suivante. Le découvreur nous a laissé un récit sobre et objectif de son expédition, au cours de son journal " en forme de lettre ". Il y décrit les principaux événements de son séjour dans l'Ouest, depuis son départ de Michillimakinac, le 20 juillet 1738 jusqu'en mai 1739. Le voyage aux Mandannes apparaît donc comme partie intégrante de l'ensemble de ses explorations et non comme un incident isolé. Il semble clair cependant, tant par les préparatifs dont elle fut l'objet que par les effectifs mis en jeu, que cette expédition prenait dans la pensée de La Vérendrye une importance particulière. Ce fut d'ailleurs le seul grand voyage de reconnaissance qu'il fit luimême, en dehors des courses routinières entre ses postes et les grands centres de Michillimakinac et de Montréal. En effet les autres expéditions ou explorations furent laissées à ses fils ou à son neveu Christophe Dufrost de la Jemmeraye. Quelle fut donc la raison de ce long et pénible voyage vers cette tribu qu'on lui avait dépeinte sous des traits si flatteurs?

Afin de mieux comprendre le sens du voyage aux Mandannes, il faut envisager l'œuvre de la Vérendrye dans son ensemble et ne pas dissocier ses essais de découverte de son effort de pénétration et de prise de possession. Cette distinction, qui donne une fausse perspective, nous est venue de Benjamin Sulte ${ }^{1}$ tout d'abord. Elle a été reprise par d'autres auteurs, et notamment par Burpee dans son Introduction à la compilation de documents sur La Vérendrye, qu'a publiée la Société Champlain. Selon Sulte et Burpee, La Vérendrye, se trouvant

1. Sulte, Benjamin, Histoire des Canadiens français, Vol 6. - Relations des Jesuites, (Thwaites) vol. 68 p. 287. 
au Lac Népigon, aurait spontanément caressé le rêve de découvrir la route qui conduisait à la Mer de l'Ouest. Il aurait interrogé les Sauvages des environs sur l'existence d'un cours d'eau allant vers l'Ouest. Il aurait recueilli, durant l'hiver de 1727-1728, tous les renseignements possibles et aurait préparé un mémoire en conséquence. Durant l'été de 1728, se trouvant à Michillimakinac, il y aurait rencontré le Père de Gonor, de retour du pays des Sioux, et lui aurait confié ce mémoire, comme on ferait à un personnage influent, l'enjoignant de l'appuyer pour se faire nommer ccmmandant de l'expédition de découverte. Le Père de Gonor aurait lui-même ajouté ses remarques à celles de La Vérendrye et aurait présenté le tout à Beauharnois à Québec, sous le titre: "Relation d'une grande rivière qui a flux et reflux, présentée par le Père de Gonor, jésuite, missionnaire des Sioux, le 3 novembre 1728 "). Grâce au Père de Gonor La Vérendrye aurait été alors n.ommé commandant de l'expédition, en 1730.

Il y a dans cette version, sinon beaucoup de fantaisie, au moins des points diffisiles à concilier avec la marche générale des événements. Soulignons tout d'abord que La Vérendrye avait demandé, en 1724 et de nouveau en 1726, de passer en France. M. de Vaudreuil, écrivant au Ministre en octobre 1724, demandait un congé pour M. La Vérendrye et trois autres gentilshommes canadiens, pour passer en France où, disait-il " ces Messieurs m'ont représenté y avoir des affaires indispensables ". Même demande au Ministre à l'automne de 1726, cette fois par M. de Beauharnois: " J'ai eu l'honneur de vous marquer dans une de mes lettres du 28 septembre dernier, à l'occasion des congés que j'ai remis aux Sieurs de la Vérendrie (sic), Celeron et Duqué, que ces officiers n'ayant pu s'en servir cette année, espèrent que vous voudrez bien, Monseigneur, leur permettre de s'en servir l'année prochaine $^{2}$ ).

Remarquons que Vaudreuil était mort en 1725 et Beauharnois son successeur, venait de débarquer à Québec, en 1726. Il apportait probablement des instructions précises de la Cour de France sur la découverte de la Mer de l'Ouest. Le projet, on le sait, avait été repris sous la Régence, surtout après la perte de la Baie d'Hudson. Le Père Charlevoix, affirme qu'en 1720 un homme avait été recommandé pour poursuivre la découverte et qu'on suggérait de lui donner un effectif

2. AC., Correspondance Générale; vol. 46, p. 103; vol. 48, p. 49. 
de 50 hommes, mais que le Régent avait décidé de l'envoyer, lui, pour enquêter ${ }^{3}$. En effet le Jésuite fit son voyage en 1721 et présenta son rapport en 1723. On connait ses deux principales recommandations: remonter le Missouri pour parvenir à la Mer de l'Ouest et établir une mission chez les Sioux, afin de faciliter le voyage. Vaudreuil abondait dans le même sens. Il avait fait rétablir le poste de Michillimakinac et avait envoyé de la Noue en 1716 avec instruction de construire trois postes: Kaministiquia, Lac la Pluie et Lac des Bois ${ }^{4}$.

Immédiatement après son arrivée, en juin 1727, Beauharnois organisa la "Société des Sioux" et chargea M. dé Boucherville de construire le Fort Beauharnois, sur le lac Pépin ${ }^{5}$. M. de Boucherville s'était fait accompagner des Pères Guignas et de Gonor, jésuites. Où l'on voit que les recommandations du Père Charlevoix n'étaient pas restées lettre morte. Sur ces entrefaites on confia à La Vérendrye, ce qu'on appelait " les Postes du Nord ". Comme il n'existe absolument aucune instruction ni lettre de la part de Beauharnois à La Vérendrye, force est bien d'en rester à des conjectures sur la nature exacte de la mission de la Vérendrye. Les "Postes du Nord" comprenaient Kaministiquia, situé à l'embouchure de la rivière du même nom, à peu près où est le Fort William d'aujourd'hui; Michipicoton, situé dans la Baje de ce nom, sur la rive nord du Lac Supérieur et celui du lac Népigon. Il est bien permis de supposer que ce fut Beauharnois qui offrit le poste à La Vérendrye et que dans la pensée du gouverneur, ce serait comme un pendant ou un complément de celui des Sioux. Il est alors facile de comprendre que Beauharnois ait recommandé et à La Vérendrye et aux missionnaires du poste des Sioux, de recueillir tous les renseignements que pourraient leur fournir les Sauvages sur la Mer de l'Ouest. Beauharnois s'engagea-t-il visà-vis de la Vérendrye? Rien n'empêche de croire qu'il lui ait fait un semblant de promesse, car le découvreur lui dira, dans le Mémoire que nous avons de lui, en date du printemps de 1730: " N'ayant rien négligé, Monsieur, depuis l'automne dernier que je suis arrivé commandant au Poste du Nord pour avoir le plus qu'il me serait possible de connaissance sur le beau et très grand fleuve qui descend

3. MARGRy, Pierre, Découvertes et établissements des franşais dans l'Ouest et dans le sud de l'A mérique septentrionale (1614-1754). Paris 1888, vol. 6, p. 521.

4. Ibidem, p. 504.

5. Ibidem, p. 547. 
droit au couchant du soleil, et la route qu'il faut tenir pour s'y rendre, j'ai eu soin de m'assurer d'un Sauvage capable d'y conduire un convoi en cas que sous le bon plaisir de Sa Majesté, vous vouliez bien m'honorer de vos ordres pour en faire la découverte " ${ }^{6}$.

A l'automne de 1730 Beauharnois et Gilles Hocquart écrivaient à Maurepas en ces termes: "Le Sieur de La Vérenderie (sic) dont il vous a été adressé des mémoires touchant la découverte de la Mer de l'Ouest, nous a proposé en conséquence de cette découverte d'aller faire un établissement sur les bords du lac Ounipigon, 550 lieus au dessous du poste de Gamanastigoya, d'où il vient de commander, et d'où il a acquis les lumières qu'il parait avoir sur la découverte de la Mer de l'Ouest. Cet officier a fondé son entreprise sur le rapport que les sauvages lui ont fait, que, de l'établissement proposé au Lac Ouinipigon, il n'y avait que dix journées courant à l'ouest pour se rendre à la mer de ce nom et quinze ou vingt en courant à l'Ouest Nord-Ouest ") 7 .

Il était exact de dire que La Vérendrye avait proposé de faire un établissement au lac Ouinipigon et de s'en servir incidemment comme d'un pied-à-terre pour la découverte de la Mer de l'Ouest. Mais il s'agissait, dans la pensée du découvreur, d'une fondation permanente. Il disait: " Les Cris sont en commerce aver les Anglais, où ils sont interprétés par les gens des terres, et il est naturel qu'ils y parlent du projet d'avoir des Français chez eux, et qu'ils donnent les mêmes connaissances qu'ils ont données ici. L'Anglais a tout intérêt à nous prévenir et si on en donne le temps il ne perdra pas l'occasion de le faire ") 8 .

Pourquoi, se demandera-t-on, Beauharnois voulait-il faire croire à Maurepas qu'il s'agissait d'un établissement temporaire, et « en conséquence de la découverte "? Pour la raison très simple que le Ministre Maurepas était un homme de science grandement intéressé par les découvertes et les explorations. On sait qu'il avait équipé, à peu près vers le même temps, des expéditions de découverte au pôle nord et à l'équateur. Il se constituait le protecteur des hommes de science: géomètres, astronomes, ingénieurs et autres. Ce fut lui qui appuya des savants comme Clairault, Maupertuis, Gobin, La Con-

6. Bunpes, Lawrence J., Journals and letters of Pierre Gaultier de Varennes de la Verendrye and his sons. Toronto, THE CHAMPLAIN SOCIETY, 1927, p. 47.

7. BurpeE, op. cit., p. 66.

8. Burpee, op. cit., p. 61 . 
damine etc. Il voulait que la marine servît la science et que la science servît la marine ${ }^{9}$. Dès le voyage de Charlevoix l'aspect scientifique de l'exploration et de la découverte de la Mer de l'Ouest l'intéressait. Et le Père Charlevoix était lui-même un savant authentique que le côté scientifique de l'entreprise intéressait aussi. Nous savons comment le Père jugea les projets de La Vérendrye: " J'estime assez inutiles les établissements dont parle cet officier, s'il entend parler autre chose que des entrepôts pour passer l'hiver quand on se sera entièrement déterminé à la route qu'on doit tenir. Ces établissements seraient de petite dépense, tireraient la découverte en longueur et pourraient dégénérer en traite des pəlleterie... ". ${ }^{10}$

Sur ce, Beauharnois et Hocquart se hâtèrent de répondre qu'il ne s'agissait précisément que d'entrepôts de fortune: "Lorsque l'cfficier, chargé de l'entreprise de la découverte de la Mer de l'Ouest, a parlé dans ses mémoires de faire un établissement au lac Ouinipigon, ou dans les endroits où il serait obligé d'hiverner, il n'a pas entendu parler d'autre chose que d'un entrepôt à pouvoir se réfugier, et afin d'être à portée d'en faire connaissance avec les Assinibosls ou autres sauvages capables de lui donner des lumières. L'on ne doit pas regarder cet établissement ou entrepôt que comme un objet qui n'a d'autre but que celui de dégénérer en traite des pelleteries ". ${ }^{11}$

La Vérendrye n'avait certainement pas l'intention de bâtir des entrepôts de fortune. Il avait risqué tout son avoir et s'était endetté fortement, il avait amené avec lui ses fils et son neveu; toutes choses qui démontrent qu'jl s'agissait d'une entreprise de longue haleine. Il le laissait d'ailleurs entendre clairement dans sa première lettre à Maurepas, écrite de Michillimakinac, le ler août 1731, alors qu'il était en route vers ses quartiers généraux de Kaministiquia : "Sur les mémoires que j'ai eu l'honneur de présenter à Monsieur le Marquis Beauharnois notre Général, et qu'il a envoyés à Votre Grandeur il m'a fait l'honneur de me détacher pour m'envoyer établir le lac Ouinipigon avec cinquante hommes et un missionnaire; j'aurai celui de l'informer l'année prochaine bien exactement de toutes les particularités de mon voyage; s'il juge à propos de m'envoyer pénétrer jusque

9. La Nouvelle Biographie Générale, article Maurepas, tome 34; cité par MorTON: " $A$ History of the Canadian West ", p. 170.

10. Burpee, op. cit., p. 75.

11. Burpee, op. cit., p. 83. 
dans l'Ouest, je serai toujours prêt à partir avec mon neveu La Jemmeraye, qui est mon second et mes trois enfants que j'ai ici avec moi ". " Je prends la liberté de représenter à Votre Grandeur que dans l'entreprise que je fais, je ne cherche qu'à porter le nom de Sa Majesté et ses armes, dans une grande étendue de pays inconnus, d'agrandir la colonje et d'y augmenter le commerce "). Et La Vérendrye demande ensuite qu'on lui accorde, pour cinq ans, les postes du nord, Gamanistigoyas et le Nipigon, exempts de congé, pour servir d'entrepôts à son entreprise, afin d'y laisser hommes, vivres et canots ${ }^{12}$.

On connaît la suite. La Vérendrye établit ou fit établir le fort Saint-Pierre sur la Rivière La Pluie, le fort Saint-Charles au Lac des Bois et le fort Maurepas sur la rivière Winnipeg. Ces établissements causèrent bien des soucis au commandant. Vaudreuil avait dit en 1719: " La difficulté de conserver les tribus de l'Ouest en paix, est inconcevable " 13 . Mais outre ces querelles d'Indiens, il y avait la question des distances et les difficultés du ravitaillement. En deux ou trois occasions l'arrivée tardive des marchandises fit manquer la traite de l'année. Puis il fallait compter avec les exigences des fournisseurs; car La Vérendrye avait dû accepter de solder lui-même tous les frais de l'entreprise. Il est étrange, pour dire le moins, que des gens comme Maurepas et Charlevoix aient pu souhaiter une marche rapide à travers un pays nouveau, et s'imaginer en même temps qu'une pareille expédition pât se faire entièrement aux frais de l'explorateur.

Suivre La Vérendrye au cours des années qui précédèrent son voyage aux Mandannes en 1738 nous entraînerait trop loin. En plus des difficultés que nous venons de signaler, il eut la douleur de perdre son fils aîné, son missionnaire et dix-huit de ses hommes massacrés en juin 1736 par les Sioux des prairies. Cet incident tragique non seulement diminuait ses effectifs, mais menaçait de causer une guerre nouvelle entre Sioux et Assiniboines et Cris, guerre qui aurait compromis toute l'œuvre de prise de possession et de colonisation future. Beauharnois invoquera cet incident pour expliquer la lenteur et le retard dans cette œuvre de découverte à laquelle Maurepas poussait constamment. Le neveu de La Vérendrye, Christophe Dufrost de la Jemmeraye mourut la même année.

12. BURPeE, op. cit., p. 70.

13. Vaudreuil au Conseil de la Marine, 28 octobre 1719. Cité par Parkman, * A Half Century of Conflict, vol. 2, p. 335. 
Nous sommes donc en 1738 et La Vérendrye est rendu à Michillimakinac, en route pour le pays des Mandannes. Durant les années précédentes il a pénétré dans le pays et y a noué des alliances avec les sauvages qui vont maintenant lui venir en aide admirablement. Il passera d'une tribu à l'autre sans aucune molestation. Son but est toujours de se gagner l'amitié des Indiens et d'établir, dans les diverses régions, des forts destinés à devenir autant de bastions de l'influence française. En octobre 1735 Beauharnois écrira à Maurepas que le Sieur de La Vérendrye continue ses travaux de prise de possession et que son " fils doit inviter les Assiniboils à les accompagner chez les Ouatchipouennes " (Mandannes); l'explorateur construira un fort proche de cette nation et il s'assurera du caractère de ces sauvages par les Assiniboils. Il doit aussi contruire une grande pirogue pour descendre le fleuve qui conduit chez ces Ouatchipouennes ${ }^{14}$.

De l'ensemble des lettres et des mouvements de La Vérendrye il ressort clairement qu'il était à la recherche du Missouri et qu'il voulait l'atteindre par le nord. Il avait, surtout vers 1736 , l'idée à peu près exacte de la route à sujvre. Ces notions, on peut dire qu'il les eut dès les débuts; et ce serait une erreur de croire que La Vérendrye voulut découvrir la Mer de l'Ouest par le lac Winnipeg. Les Sauvages, il est vrai, lui tracèrent une carte du lac Winnipeg, qui indique une rivière à remonter et laquelle, après un certain nombre de jours de canot, était censée couler vers l'ouest; mais il est clair encore là que le découvreur y vit tout au plus une rivière qui conduirait à celle du Missouri, déjà désigné depuis 1717 par le Sieur Hébert, ordonnateur de la Louisiane comme la route la plus propice vers la mer tant cherchée. ${ }^{15}$ Mais on le sait, un conflit de juridiction existait entre la Louisiane et le Canada au sujet de ces contrées éloignées. Vaudreuil se plaint des ambitions de la Louisiane sur les pays à l'ouest des Illinois et même sur le Wisconsin. Il est possible qu'afin d'éviter toute querelle, les intendants de la Nouvelle-France, aient poussé la découverte par le nord en remontant le Missouri.

Ce voyage de La Vérendrye nous en laisse nettement l'impression: il consacre une politique et une situation de fait. Le découvreur procède avec diligence et précision. Parti de Michimillimakinac le 20

14. BURPee, op. cit., p. 204.

15. Margry, vol. VI, p. 187. 
juillet, il est à Kaministiquia le 5 août. Il en repart le lendemain et s'arrête au fort Saint-Pierre sur la Rivière la Pluie et, de là, se rend directement au fort Saint-Charles, devenu son centre d'opération. Il en repart le 11 septembre après s'être assuré " que tout était en bon ordre, tant pour le fort que pour mon départ ". Accompagné de ses deux fils, François et Louis-Joseph, il arrive au Fort Maurepas sur la rivière Winnipeg le 22 septembre. Deux jours plus tard il est à ce qu'il appelle " La Fourche des Assiniboils " c'est-à-dire à l'embouchure de l'Assiniboine, là où s'élèvent aujourd'bui les villes de Winnipeg et Saint-Boniface. Il repart le 26 septembre, suivant à pied la direction de l'Assiniboine, vers le Couchant. Il s'arrête finalement le 2 octobre à l'endroit du portage qui conduit aux lacs Manitoba et Winnipegosis et aux Anglais de la Baie d'Hudson. C'est là qu'il fera édifier le Fort La Reine, pour canaliser le commerce qui lui échappait.

Dans tous les lieux qu'il visite au cours de son voyage, La Vérendrye réunit les sauvages amis des Français et les engage à rester fidèles à leur alliance. Il leur parle de leur père, le Gouverneur, qu'il a vu à Québec et il leur recommande de protéger les Français qui sont parmi eux et de leur fournir les fourrures dont ils ont besoin. Le commandant se livre à une espèce de revue. Et on peut facilement constater le progrès qu'il a accompli. Son œuvre d'établissement est maintenant solide et bien ordonnée. Il a conquis la confiance et l'amitié des Sauvages et lui et ses fils connaissent maintenant le pays à la perfection. Voici donc La Vérendrye en état d'entreprendre le voyage d'exploration qu'il eût pu faire en 1736 , n'eût été l'horrible massacre de juin. Le voyage aux pays des Mandannes fait partie d'un projet de découverte et de la découverte de la mer de l'Ouest, même si La Vérendrye a pu s'enquérir si oui ou non il était possible de jeter en ces régions des établissements permanents. Chose certaine le Père Aulneau écrira du Fort Saint-Charles en 1736 qu'il a été envoyé pour se rendre chez les Mandannes " pour lesquels mes supérieurs me font faire ce long voyage ".$^{16}$

Voilà les raisons pour lesquelles La Vérendrye fera le voyage et voilà en quelles conditions il l'entreprit.

Antoine D'EschambaulT

16. Lettre du Père Aulneau, Fort Saint-Charles, 30 avril 1736. 